\title{
3-D Fusion of Biplane Angiography and Intravascular Ultrasound for Accurate Visualization and Volumetry
}

\author{
Andreas Wahle ${ }^{1}$, Guido P. M. Prause ${ }^{1,3}$, Steven C. DeJong ${ }^{2}$, and Milan Sonka ${ }^{1}$ \\ 1 The University of Iowa, Department of Electrical and Computer Engineering \\ Iowa City, IA 52242, USA \\ \{andreas-wahle,milan-sonka\}@uiowa.edu \\ 2 The University of Iowa, Department of Internal Medicine \\ Iowa City, IA 52242, USA \\ 3 MeVis Institute at the University of Bremen \\ D-28359 Bremen, Germany
}

\begin{abstract}
Coronary angiography delivers accurate information about the vessel topology and shape, but only limited data concerning the vessel cross-section. Intravascular ultrasound provides detailed information about the cross-sectional shape as well as the composition of vessel wall and plaque, but fails to consider the geometric relationships between adjacent images. In this paper, we present a new approach for combination of both methods to allow accurate assessment of coronary arteries regarding both longitudinal and cross-sectional dimensions.
\end{abstract}

\section{Introduction}

During the last decades, quantitative analyses from selective coronary contrast angiographic images have been established as de-facto standard for the diagnosis of coronary artery disease. Well-established systems for single-plane analysis of local stenoses [1-3] have been complemented by accurate spatial reconstructions from biplane angiograms, allowing assessment of complex diffuse disease affecting the entire coronary artery system [4-6]. However, the major limitation of X-ray angiography is its restriction to the inner lumen. The cross-sectional shape is mostly approximated by elliptical contours, and the wall thickness as well as the plaque composition cannot be determined at all.

Recently, intravascular ultrasound (IVUS) of the coronary arteries evolved as an additional method in cardiovascular diagnosis. A catheter with an ultrasonic transducer in its tip is placed at the distal end of the desired vessel segment and pulled back with an approximately constant speed during imaging. The luminal cross-sections can be determined as well as the wall thickness, and even the composition of the plaque [7-9]. On the other hand, IVUS is not able to consider vessel curvature and torsion when assigning the detected plaque to specific locations. Spatial reconstructions as performed up to now by straight stacking of the images are geometrically incorrect and thus do not allow proper 
volumetric analyses. For a correct reconstruction, the following effects have to be dealt with [10-12]:

- Due to vessel curvature, the IVUS slices are not parallel. Thus, volume fragments at the inner side of the vessel related to its curvature will be overestimated and fragments on the outer side underestimated during volumetric analysis.

- Due to vessel torsion as defined by differential geometry, the axial orientation of an ideal IVUS catheter within the vessel is no longer constant. The torsion of the catheter path is zero only if the vessel lies within a plane. Whenever the vessel moves outside of this plane, the catheter twists axially, and this rotation must be considered in the 3-D reconstruction as well.

Our solution for these problems is derived using the combination of the data obtained from coronary angiography and intravascular ultrasound to provide an exact assignment of the cross-sectional data to the vessel segment in both location and orientation. The 3-D vessel shape and topology are determined by accurate reconstruction from biplane angiograms of approximately known imaging geometry. The vessel cross-sections are extracted frorn IVUS data acquired in the same session as the angiographic images. For both tasks, our previously developed and validated systems are utilized.

\section{Methods}

\subsection{Image Acquisition and Preprocessing}

Placement and pullback of the IVUS catheter is usually supervised by fluoroscopy, thus all required data can be obtained in a single step. Both angiograms and IVUS images have to be digitized since they are usually acquired on conventional media like cine film and S-VHS tapes. It is desirable to avoid this step by direct digital access to the imaging devices in standardized formats in the near future.

While the IVUS sequence reflects the entire pullback, angiograms are taken only at the start of the pullback, eventually an additional pair at the end. A low amount of radio-opaque contrast dye may be injected to allow an identification of the vessel lumen without covering the IVUS catheter itself. A constant supervision of the catheter tip movement by angiography as proposed by Evans et al. [11] is not acceptable in clinical applications, due to physical limitations of the imaging device and the additional X-ray exposure of the patient.

The angiograms are corrected for geometric distortions like the pincushion and sigmoidal effects [13]. Further visual manipulations, e.g. edge enhancement by unsharp masking, adaptive histogram equalization, zooming, etc., can be performed to allow a better identification of the catheter (Fig. 1).

\subsection{Generation of Spatial IVUS Frames}

In this section, the basis for the geometrically correct reconstruction of the IVUS images is described. For each of the 2-D images, a 3-D frame is calculated, which 

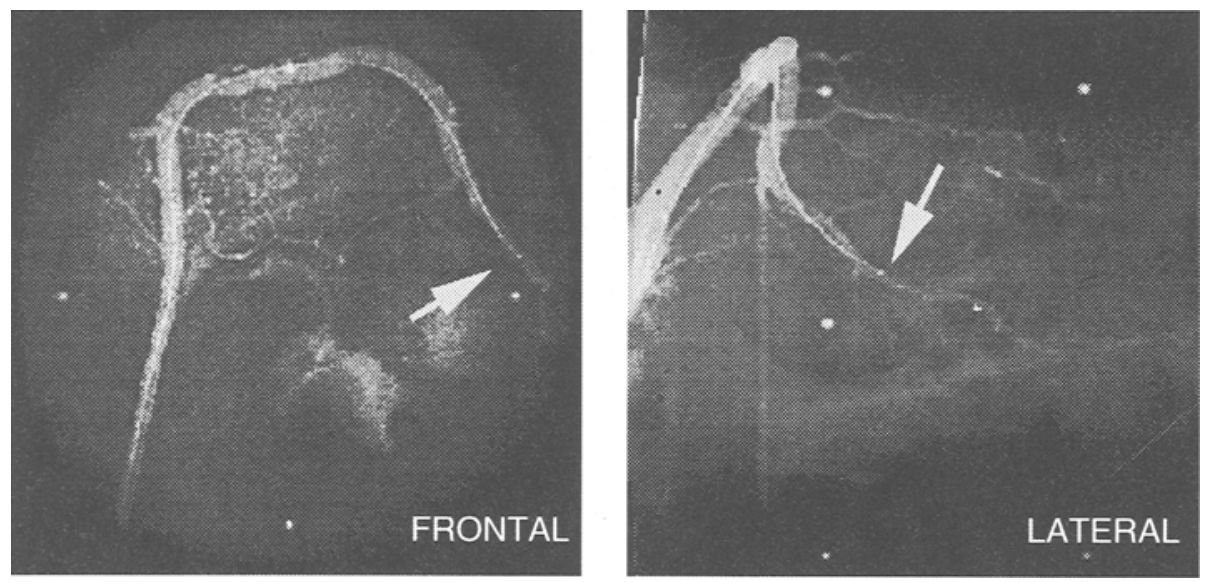

Fig. 1. Preprocessed biplane angiograms in a right coronary artery of an extracted cadaveric pig heart with inserted IVUS catheter; the arrows mark the location of the IVUS transducer at pullback start, i.e. at the most distal location.

represents a mapping function of the planar images into spatial dimensions. Once these frames are calculated, they may be filled with the plain pixel data as well as the high-level results from the segmentation. The frames are determined from the information contained in the angiograms, by extracting the path of the catheter, mapping the IVUS images to locations, and calculating their orientations.

Catheter Extraction. From the rectified angiograms, the catheter may be extracted by conventional methods for vessel detection $[2,3]$. Furthermore, we developed a new dynamic programming approach to find the correct path of the catheter even in vessels filled with contrast dye. The starting point of the pullback has to be marked in both projections, and then some proximal guide points, at least up to the end of the pullback. The catheter is detected within an adaptable region of interest (ROI) along the guide points. A recent approach combines catheter extraction and 3-D reconstruction in a single step using active contour models (3-D snakes [14]).

3-D Reconstruction of the Trajectory. The IVUS catheter is reconstructed as a single line in 3-D without cross-sectional information from the angiographic projections. A single point, identified in both projections, can be reconstructed by calculating its projection rays in 3-D from the known imaging geometry. The reconstruction algorithm as initially reported in [13] has meanwhile been replaced by the comprehensive approach as developed at the German Heart Institute of Berlin $[6,15]$ to achieve higher accuracy. For each object point $\mathrm{P}$ visible in both projections $p$, its projection rays can be calculated from the known locations of the X-ray sources $\mathrm{A}_{p}$ and the points $\mathrm{B}_{p}$, resulting from the transformation of 
the respective image points to image intensifier level. Theoretically, these rays should intersect in the original 3-D location $\mathrm{P}$ at imaging time, provided that the assumed imaging geometry is correct and both projection points have been identified accurately. Since this is hard to ensure, an approximation has to be performed to find the location of the point to be reconstructed. This is done by using the weighted mean of those points $\mathrm{Q}_{p}$ on both rays $\left(\mathrm{A}_{p}, \mathrm{~B}_{p}\right)$ that mark the nearest distance between them (Fig. 2).

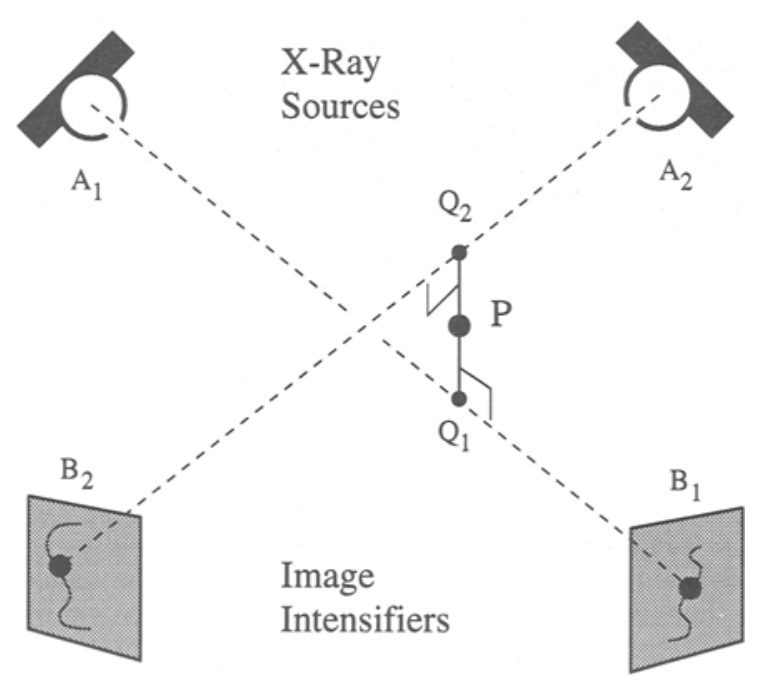

Fig. 2. Principle of point reconstruction from two projections.

After estimating the initial geometry from the parameters given by the gantry, the assumed imaging geometry is refined using $n$ unique reference points in the angiograms by an approximation method minimizing the reconstruction error $\left|\mathbf{Q}_{1}(i)-\mathbf{Q}_{2}(i)\right|$ over all points $0 \leq i<n$.

Furthermore, the ray bundles resulting from the catheter extraction have to be matched, i.e. those pairs of elements have to be found that correspond in both projections. The initial discrete mapping algorithm as introduced by Parker et al. [16], which assigns pairs of 2-D elements within a cost matrix to yield the optimum combinations, has been extended to interpolate intermediate elements for determination of a more accurate correspondence. Further enhancements were done to stabilize the cost matrix approach when applied to larger vessel segments by smoothing in the correspondence domain, mainly to eliminate distortions from local roughness.

Mapping of the Image Locations. In the following step, the frame of each IVUS image has to be assigned to a specific location along the pullback path. Due to the fact that a continuous supervision of the catheter pullback is not 
available, its speed is assumed to be constant, which is approximately true if a mechanical pullback device is used [10]. Since the length from pullback start to any location on the path can be determined accurately $[6,15]$, the location of each frame can be determined from its timestamp. If - in addition to the start image - another biplane angiographic pair exists for the end of the pullback, this information can be used to calculate the real mean pullback speed for a better assignment.

Calculation of the Catheter Twist. After assigning the frames to their locations, the spatial orientation has to be determined for each of them. For this purpose, a sequential triangulation was developed to calculate the relative twist between adjacent IVUS images (Fig. 3). This is an approximation to the Frenet-Serret rules, which cannot directly be used in this case since only a set of discrete points ist available from the reconstructed pullback path. For each pair of adjacent IVUS images, their tangent vectors are determined using the three surrounding consecutive points $\left(\mathrm{P}_{i}, \mathrm{P}_{i+1}, \mathrm{P}_{i+2}\right)$ along the catheter path. The arc through these three points is a part of the circumscribed circle, whose center is determined as the intersection of the perpendicular bisectors of the tangent vectors. The orientation of frame $i+1$ is determined by rotating frame $i$ by the enclosed angle $\alpha_{i}$ around the normal vector $\mathrm{n}_{i}$ of this triangle.

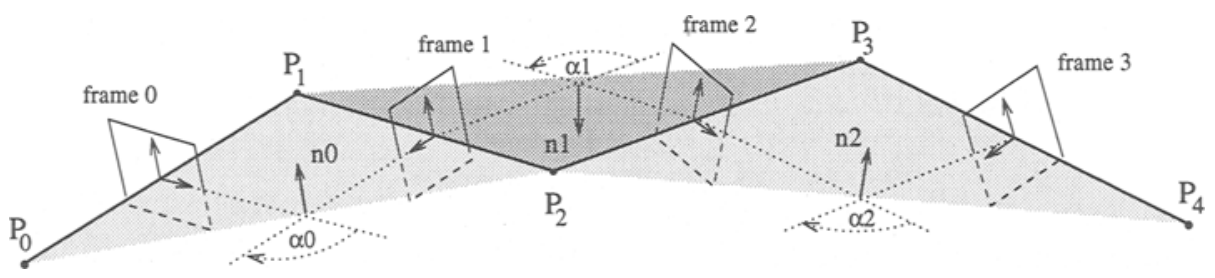

Fig. 3. Calculation of the relative twist by sequential triangulation.

Although the relative twist can be calculated, the absolute orientation remains ambiguous. One way to achieve a correct overall match of the IVUS and angiography data is to use anatomic landmarks like branching vessels. Unfortunately, the locations and orientations of branches frequently cannot be determined accurately enough both in angiographic and IVUS images [13]. Algorithms to solve this problem automatically by using additional information and physical constraints are under development. Currently, the absolute orientation is determined by user interaction.

Fig. 4 shows the reconstructed frames from the angiograms of Fig. 1. The longitudinally resampled slice of the geometrically correct reconstructed IVUS cube corresponds to the orientation of the frontal angiogram. 

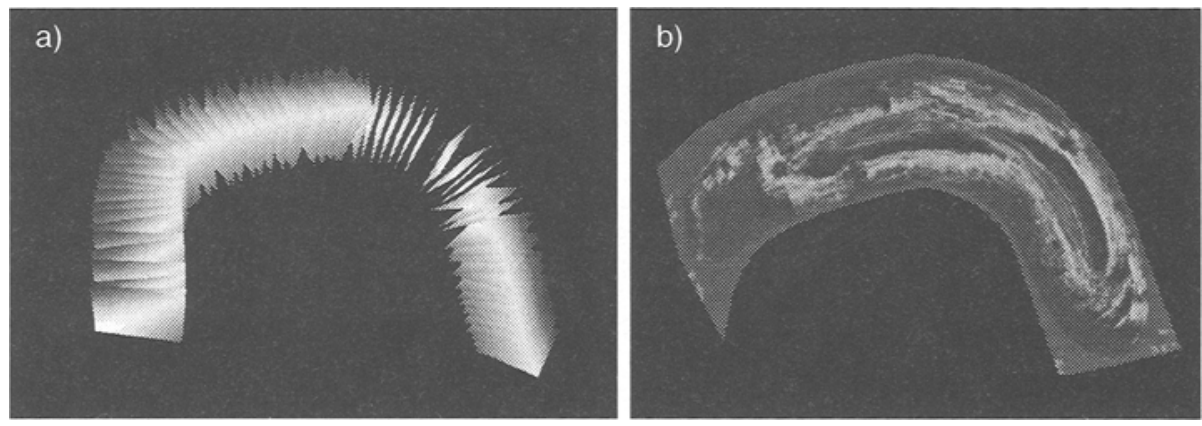

Fig. 4. Spatially correct IVUS reconstruction: a) Calculated IVUS frames with colorcoded corners; b) after mapping the pixel data into the frames, a longitudinal slice of the descending arc has been extracted.

\subsection{IVUS Segmentation}

Our method as earlier described in [7] automatically identifies the plaque/media interface (internal lamina, internal wall border), the media/adventitia interface (external lamina, external wall border), and the plaque/lumen interface (plaque border). Due to the use of a-priori information about 2-D and 3-D anatomy of coronary vessels and ultrasound imaging physics, the method can automatically determine vessel wall morphology and plaque volumes. The two key aspects of the approach are as follows:

1. Graph searching is utilized to identify globally optimal borders.

2. A-priori information is incorporated into the border detection process through the computation of local cost values.

In particular, to identify the position of the internal and external wall borders, the method searches for edge triplets representing the leading and trailing edges of the laminae echoes. The interactively defined elliptical shape of the ROI serves as a model of the preferred vessel shape. Knowledge of the vessel wall thickness is also used to constrain the search for the external and internal wall borders.

To facilitate a fully automated 3-D analysis of IVUS pullback image sequences, the region of interest in which the borders are determined must be automatically identified when the 3-D border detection is performed. The 3$D$ border detection algorithm was designed to use contextual information for the estimation of the ROI size and position changes in the successive frames. The general steps of the 3-D IVUS segmentation algorithm are as follows: A single elliptic region of interest is interactively identified in the first image of the pullback sequence. Inside the ROI, internal and external wall, and plaque borders are automatically identified using the graph-search based segmentation approach. In subsequent images, current ROIs are automatically determined from the computer-detected borders in the preceding images.

Fig. 5 shows an example of automated inner and outer vessel wall detection in an IVUS image of the sequence for the angiograms in Fig. 1 with our algorithm. 


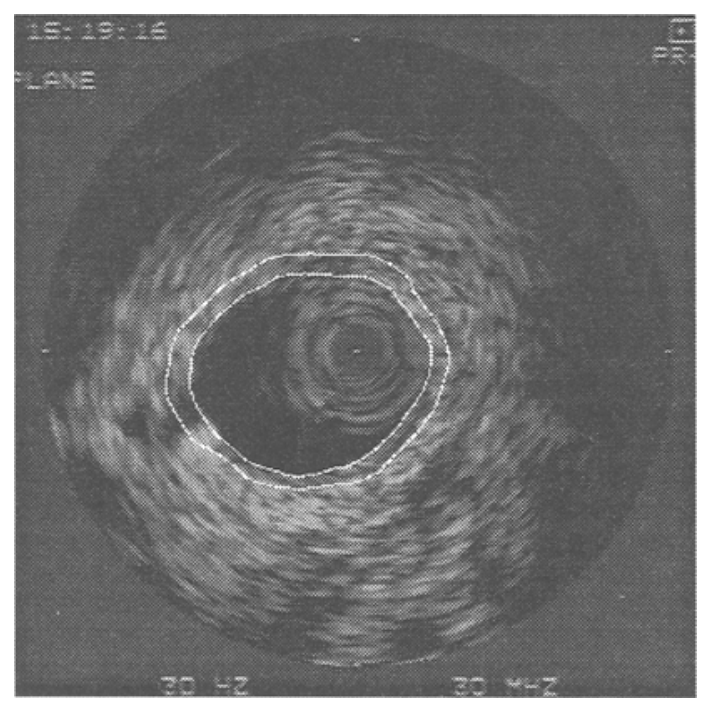

Fig. 5. Segmented IVUS image with inner and outer borders of the vessel wall.

\subsection{3-D Visualization}

After mapping of the pixel data into 3-D space, the volume cube may be analyzed with the conventional methods for volume rendering (Fig. 4b). A detailed surface model of the inner and outer borders of the vessel wall, as well as the plaque, can be generated after segmentation. This model is represented together with the spatially correct locations of the angiograms and the X-ray sources in a VRML-2.0 world, thus allowing visualizations platform-independently by any appropriate viewer (Fig. 6).

\section{Results}

\subsection{Frame Mapping}

For validation of the frame generation, studies in both phantoms and cadaveric pig hearts were performed. Analyses in helical phantoms have shown a good match of the measured and the analytically determined twist [12]. The predicted axial rotation of the IVUS images in helices of different displacements per revolution showed high correlations of $r=0.99$, but also some overestimation of the real twist by $0.87-1.0 \%$ per centimeter of pullback.

For the in-vitro studies in right coronary arteries, with a typical pullback length of $100-120 \mathrm{~mm}$, the overall twisting range along individual pullbacks was $41.8^{\circ}$ in the least tortuous up to $118.1^{\circ}$ in the most tortuous vessel. Two of the hearts which we evaluated were supplied with metal markers (Fig. 7). Three pullbacks per heart were performed by a highly experienced person, instructed 


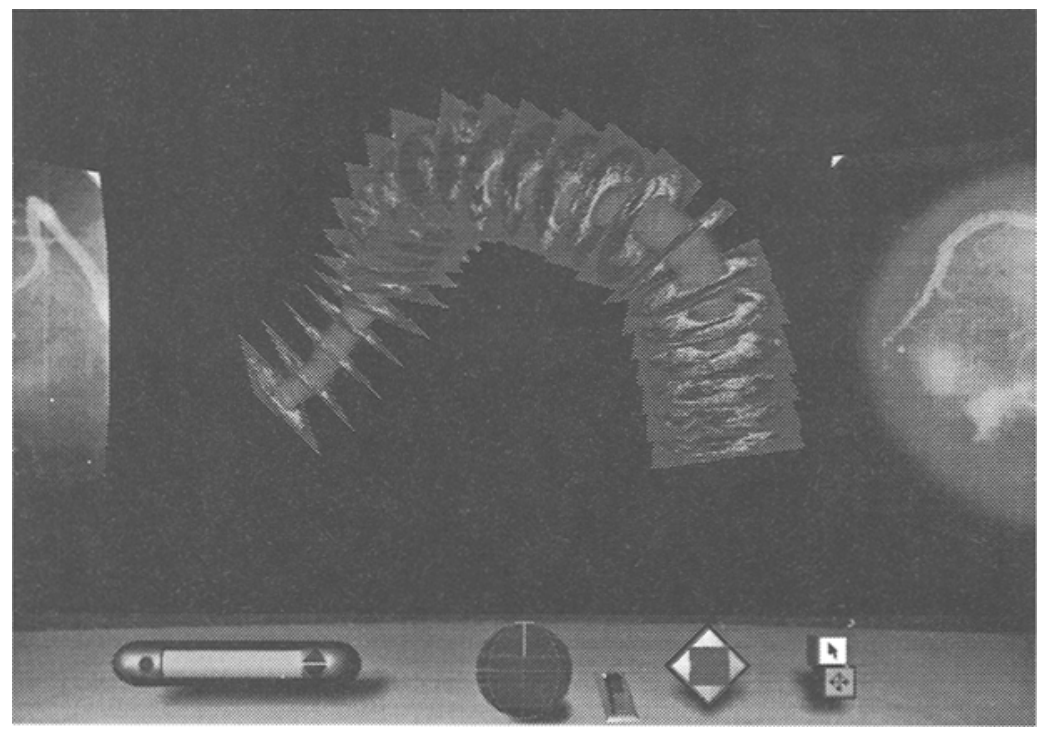

Fig. 6. VRML-2.0 model after triangulation of the segmented contours: internal and external laminae along with some IVUS images inserted according to their calculated spatial frames; X-ray sources (behind the viewpoint) and angiograms are included as well; the lower part of the scene shows the control panel of the VRML viewer.

to maintain a constant pullback speed of $1 \mathrm{~mm} / \mathrm{s}$. However, using the clips as landmarks, a pullback speed of $1.14 \pm 0.34 \mathrm{~mm} / \mathrm{s}$ resulted, which indicates that the manual pullback is significantly non-uniform. The RMS error of the predicted frame orientations over all six pullbacks was $21.96^{\circ}$ with a standard deviation of $4.87^{\circ}$. The highest deviations from mean were measured for the clips located in areas of high local twisting, where the slight mismatches in their locations are quite significant. Other important influences include intraobserver variability due to manual pullback with an RMS error of $5.01^{\circ}\left(\max .27 .5^{\circ}\right)$, as well as additional twisting of the mechanically driven catheter system due to friction and non-uniform rotation distortions. In phantom experiments, the twisting showed errors up to $57^{\circ}$ in areas of bending (curvature of $50^{\circ}$ over a segment of $15 \mathrm{~mm}$ in length). During saline flush, we could measure a twisting of up to $70^{\circ}$, presumptively due to the increased pressure.

\subsection{IVUS Segmentation}

The automated method for the detection of borders in IVUS images was validated in cadaveric hearts as well as in-vivo in single images and image pullback sequences [7]. Lumen and plaque area measurements correlated well with those determined manually by expert observers $(r=0.98, y=1.01 x+1.51 ; r=0.94$, $y=1.06 x-0.09$; respectively). Root-mean-square wall and plaque border positioning errors were under $0.15 \mathrm{~mm}$. 

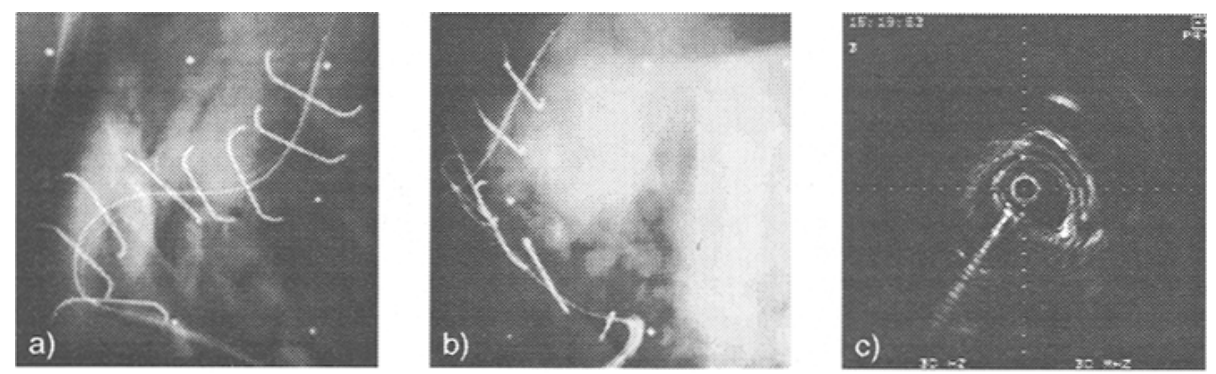

Fig. 7. Cadaveric pig heart with metal clips: a) frontal and b) lateral angiographic projections; c) IVUS image with peak caused by a clip.

\section{Conclusion}

Despite the fact that the manual pullback cannot be assumed to be of constant speed, the matching algorithms showed a very good accuracy. However, the use of an automatic pullback device is strongly recommended. Possible sources of the errors in the frame orientations, aside the manual pullback, were found in systematic torsions within the catheter system. These effects may be avoided or at least reduced by using solid-state catheter systems with no rotating parts.

In conclusion, our system provides high accuracy in 3-D reconstruction of the vessel topology, and a spatially correct assignment of plaque and wall data as delivered by the IVUS segmentation.

\section{Acknowledgments}

This work has been supported in part by grants $\operatorname{Pr} 507 / 1-2$ and Wa 1280/1-1 of the Deutsche Forschungsgemeinschaft, Germany, and by grants IA-94-GS-65 and IA-96-GS-42 of the American Heart Association, Iowa Affiliate.

\section{References}

1. Kirkeeide R. L., Fung P., Smalling R. W., and Gould K. L., Automated evaluation of vessel diameter from arteriograms, in: Proc. Computers in Cardiology 1982, Seattle WA. IEEE-CS Press, Los Alamitos CA (1982) 215-218.

2. Beier J., Oswald H., Sauer H. U., and Fleck E., Accuracy of measurement in quantitative coronary angiography (QCA), in: Lemke H. U., Rhodes M. L., Jaffe C. C., and Felix R. (eds.), Computer Assisted Radiology (CAR '91). Springer, Berlin/New York (1991) 721-726.

3. Sonka M., Winniford M. D., and Collins S. M., Robust simultaneous detection of coronary borders in complex images. IEEE Transactions on Medical Imaging 14 (1995) 151-161.

4. Guggenheim N., Doriot P. A., Dorsaz P. A., Descouts P., and Rutishauser W., Spatial reconstruction of coronary arteries from angiographic images. Physics in Medicine and Biology 36 (1991) 99-110. 
5. Seiler C., Kirkeeide R. L., and Gould K. L., Basic structure-function relations of the epicardial coronary vascular tree; basis of quantitative coronary arteriography for diffuse coronary artery disease. Circulation 85 (1992) 1987-2003.

6. Wahle A., Wellnhofer E., Mugaragu I., Sauer H. U., Oswald H., and Fleck E., Assessment of diffuse coronary artery disease by quantitative analysis of coronary morphology based upon 3-D reconstruction from biplane angiograms. IEEE Transactions on Medical Imaging 14 (1995) 230-241.

7. Sonka M., Zhang X., Siebes M., Bissing M. S., DeJong S. C., Collins S. M., and McKay C. R., Segmentation of intravascular ultrasound images: A knowledge-based approach. IEEE Transactions on Medical Imaging 14 (1995) 719-732.

8. Dijkstra J., Wahle A., Koning G., Reiber J. H. C., and Sonka M., Quantitative coronary ultrasound: State of the art, in: Reiber J. H. C. and van der Wall E. E. (eds.), What's New in Cardiovascular Imaging? Vol. 204 of Developments in Cardiovascular Medicine, Kluwer, Dordrecht (1998) 79-94.

9. Sonka M. and Zhang X., Assessment of plaque composition using intravascular ultrasound, in: Reiber J. H. C. and van der Wall E. E. (eds.), What's New in Cardiovascular Imaging? Vol. 204 of Developments in Cardiovascular Medicine, Kluwer, Dordrecht (1998) 183-196.

10. Laban M., Oomen J. A., Slager C. J., Wentzel J. J., Krams R., Schuurbiers J. C. H., den Boer A., von Birgelen C., Serruys P. W., and de Feyter P. J., ANGUS: A new approach to three-dimensional reconstruction of coronary vessels by combined use of angiography and intravascular ultrasound, in: Proc. Computers in Cardiology 1995, Vienna AT. IEEE Press, Piscataway NJ (1995) 325-328.

11. Evans J. L., Ng K. H., Wiet S. G., Vonesh M. J., Burns W. B., Radvany M. G., Kane B. J., Davidson C. J., Roth S. I., Kramer B. L., Meyers S. N., and McPherson D. D., Accurate three-dimensional reconstruction of intravascular ultrasound data; spatially correct three-dimensional reconstructions. Circulation 93 (1996) $567-576$.

12. Prause G. P. M., DeJong S. C., McKay C. R., and Sonka M., Towards a geometrically correct $3-\mathrm{D}$ reconstruction of tortuous coronary arteries based on biplane angiography and intravascular ultrasound. International Journal of Cardiac Imaging 13 (1997) 451-462.

13. Prause G. P. M., DeJong S. C., McKay C. R., and Sonka M., Semi-automated segmentation and 3-D reconstruction of coronary trees: Biplane angiography and intravascular ultrasound data fusion, in: Proc. Medical Imaging 1996: Physiology and Function from Multidimensional Images, Newport Beach CA. Vol. 2709, SPIE, Bellingham WA (1996) 82-92.

14. Molina C., Prause G. P. M., Radeva P., and Sonka M., 3-D catheter path reconstruction from biplane angiograms, in: Proc. Medical Imaging 1998: Image Processing, San Diego CA. Vol. 3338, SPIE, Bellingham WA (1998) 504-512.

15. Wahle A., Präzise dreidimensionale Rekonstruktion von Gefäßsystemen aus biplanen angiographischen Projektionen und deren klinische Anwendung. No. 152 in Fortschritt-Berichte, Reihe Biotechnik (17), VDI Verlag, Düsseldorf (1997).

16. Parker D. L., Pope D. L., van Bree R. E., and Marshall H. W., Three-dimensional reconstruction of moving arterial beds from digital subtraction angiography. Computers and Biomedical Research 20 (1987) 166-185. 